\title{
ACCELERATED MICROSTRUCTURE IMAGING VIA CONVEX OPTIMISATION FOR REGIONS WITH MULTIPLE FIBRES $\left(\right.$ AMICO $\left._{\mathrm{X}}\right)$
}

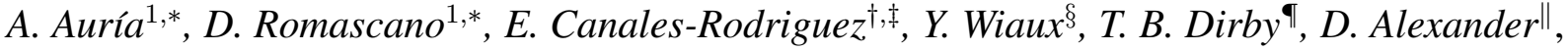 \\ J.P. Thiran,**, A. Daducci,*** \\ * LTS5, Ecole Polytechnique Fédérale de Lausanne (EPFL), Switzerland \\ ${ }^{\dagger}$ FIDMAG Germanes Hospitalaries, Barcelona, Spain \\ $\ddagger$ CIBERSAM, Spain \\ $\S$ ISSS, Heriot-Watt University, Edinburgh, United Kingdom \\ I Danish Research Centre for Magnetic Resonance, Copenhagen University Hospital, Hvidovre, Denmark \\ " CMIC, University College London, London, United Kingdom \\ ** University of Lausanne (UNIL), Lausanne, Switzerland \\ ${ }^{1}$ Both authors equally contributed to this work
}

\begin{abstract}
This paper reviews and extends our previous work to enable fast axonal diameter mapping from diffusion MRI data in the presence of multiple fibre populations within a voxel. Most of the existing microstructure imaging techniques use non-linear algorithms to fit their data models and consequently, they are computationally expensive and usually slow. Moreover, most of them assume a single axon orientation while numerous regions of the brain actually present more complex configurations, e.g. fiber crossing. We present a flexible framework, based on convex optimisation, that enables fast and accurate reconstructions of the microstructure organisation, not limited to areas where the white matter is coherently oriented. We show through numerical simulations the ability of our method to correctly estimate the microstructure features (mean axon diameter and intracellular volume fraction) in crossing regions.
\end{abstract}

Index Terms - diffusion MRI, microstructure imaging, convex optimisation.

\section{INTRODUCTION}

The challenge in diffusion Magnetic Resonance Imaging (dMRI) is to infer features of the local tissue anatomy, composition and microstructure from water displacement measurements. Water diffusion in living tissues is highly affected by its cellular organisation [1]. In particular, water does not diffuse equally in all directions in a highly ordered organ such as the brain and this property can be exploited to study the structural neural connectivity in a non-invasive way. Diffusion Tensor Imaging (DTI) [2], Diffusion Spectrum Imaging (DSI) [3], Diffusion Orientation Transform (DOT) [4] and spherical deconvolution methods $[5,6,7]$ are among a great variety of techniques developed to recover the fibre configuration from dMRI data. All the aforementioned techniques are able to estimate the fibre orientations (number and direction of the fibre populations) in a

This work is supported by the Center for Biomedical Imaging (CIBM) of the Geneva-Lausanne Universities and the EPFL, as well as the foundations Leenaards and Louis-Jeantet. voxel. However, they do not provide any information on their microstructural properties like axon diameter and density, which are related to conduction velocity [8] and play an important role in the performance of the white matter (WM) tracts. Also, the microstructure organisation of specific areas of the brain change in subjects affected by certain pathologies, such as multiple sclerosis [9]. Therefore, the study of the microstructure in vivo represents a major goal in neuroscience but also from the clinical point of view.

Most microstucture imaging techniques recover the microstructure properties by modelling the signal decay in different tissue compartments, e.g. axons, glial cells and extra-axonal space. These methods can infer not only the orientation of the main fibre populations in a voxel, but also their microstructural properties, such as the average diameter and density of the axons. For an exhaustive survey of the existing techniques in the field the reader can refer to [10]. Recently, Alexander et al. developed ActiveAx [11], which allows the estimation of orientationally-invariant indices of axon diameter and density in scan time tolerable by live human subjects. ActiveAx uses a Minimal Model of White Matter Diffusion (MMWMD) with four compartments to describe the measured dMRI signal $[11,12]$ : besides the restricted and hindered compartments previously considered by [13], the MMWMD accounts also for stationary water trapped within small structures such as glial cells as well as free water characterised by isotropic diffusion. ActiveAx was recently extended to allow axon diameter mapping also in regions with crossing fibres [14] and thus, overcome the main limitation of the majority of microstructure imaging methods which are restricted to regions with one single fibre orientation. All the techniques mentioned so far have demonstrated the practical possibility to estimate microstructural information from dMRI data and the estimated microstructural indices have been shown to agree very well with known anatomical patterns observed with histology $[11,15,12]$. However, the non-linear routines usually employed to fit these models are computationally very intensive and cause practical problems for their application in clinical studies.

Recently, Daducci et al. presented a flexible framework for Accelerated Microstructure Imaging via Convex Optimisation (AM- 
ICO) [16] to reformulate these microstructure imaging techniques as linear systems that can be solved using convex optimisation methods. The convex optimisation framework allows to include prior information about the signal, such as positivity, as long as it is formulated as a convex constraint. Besides this flexibility, convex optimisation methods are fast and many efficient numerical algorithms exist to solve them [17]. However, despite the drastic improvement in speed, the current model for AMICO is only valid in regions with one fibre population, making it inadequate for many widespread regions of the brain with multiple fibre bundles.

In this work, we extend the AMICO framework to be able to recover microstructure parameters also in regions with multiple fibre populations using fast algorithms. Numerical simulations evidence the ability of our new algorithm to recover microstructure parameters in regions with crossings, where the original AMICO formulation [16] is shown to be inadequate.

\section{DATA MODEL AND METHODS}

The reconstruction problem for microstructure features from diffusion data accounting for multiple fibres is presented here as an extension of AMICO [16]. Throughout this paper, we keep the same problem formulation as in in the original paper. To make the present article self-contained, we recall it in this section and point out the reader to [16] for further details.

The microstructure mapping problem is expressed in terms of a linear formulation, as follows:

$$
\boldsymbol{y}=\Phi \boldsymbol{x}+\eta,
$$

being $\boldsymbol{y} \in \mathbb{R}_{+}^{N_{d}}$ the vector of diffusion measurements, $\boldsymbol{x} \in \mathbb{R}_{+}^{N_{k}}$ the coefficients to be estimated and $\Phi$ the linear operator or dictionary that models the convolution operator. The AMICO framework can be applied to different microstructure imaging models, as shown in [16]. In this work we focus on extending the formulation for the ActiveAx model [11] to allow axonal diameter mapping in case of multiple fiber populations within a voxel.

The reconstruction problem is decoupled into two simpler subproblems. First, the number and orientation of the fibre populations $\boldsymbol{\mu}_{i} \in \mathbb{S}^{2}$ in each voxel is estimated. This can be achieved using any of several reconstruction methods, such as the standard Constrained Spherical Deconvolution (CSD) method [7]. Secondly, the linear operator $\Phi$ to express ActiveAx as a linear system is built from different sub-matrices:

$$
\Phi=\left[\Phi_{1}^{r}\left|\Phi_{1}^{h}\right| \ldots\left|\Phi_{M}^{r}\right| \Phi_{M}^{h}\right] .
$$

In equation (2), sub-matrices $\Phi_{i}^{r} \in \mathbb{R}^{N_{d} \times N_{r}}$ and $\Phi_{i}^{h} \in \mathbb{R}^{N_{d} \times N_{h}}$ model, respectively, the intra-axonal and extra-axonal contributions to the diffusion signal along the direction $\boldsymbol{\mu}_{i}(i=1, \ldots, M) . M$ stands for the total number of detected fibre populations in the voxel. Each atom in sub-matrices $\Phi_{i}^{r}$ models the diffusion signal corresponding to water molecules restricted within parallel cylinders of a specific diameter. Alternatively, the atoms in sub-matrices $\Phi_{i}^{h}$ describe the hindered space between the axons. $N_{r}$ and $N_{h}$ represent, respectively, the number of different axon radii and hindered environments considered to build the dictionary. The signal response matching both restricted and hindered water diffusion in a voxel is estimated using the same models and parameter set as in [16]. For further details, the reader can refer to the original manuscript and find a specific description of these models in [10].

Equation (1) is then solved as a Tikhonov-regularised least-squares problem as follows:

$$
\min _{\boldsymbol{x} \geq 0} \frac{1}{2}\|\Phi \boldsymbol{x}-\boldsymbol{y}\|_{2}^{2}+\lambda \frac{1}{2}\|\boldsymbol{x}\|_{2}^{2}
$$

where $\|\cdot\|_{2}$ is the standard $\ell_{2}$ norm and the parameter $\lambda>0$ controls the trade-off between data regularisation terms. The microstructure indices of interest defined by Alexander et al. [11] can be estimated for each individual fiber population from the recovered coefficients $\boldsymbol{x}$ by partitioning them as $\left[\boldsymbol{x}_{1}^{r}\left|\boldsymbol{x}_{1}^{h}\right| \ldots\left|\boldsymbol{x}_{M}^{r}\right| \boldsymbol{x}_{M}^{h}\right]$, corresponding to the contributions of hindered and restricted compartments from every fibre bundle. In every voxel, the intra-axonal volume fraction $\nu^{\prime}$ indicates the ratio between restricted and hindered compartments; and for each of the fibre populations $i(i=1, \ldots, M)$, the mean axon diameter $a_{i}^{\prime}$ is expressed as a weighted average of the coefficients corresponding to restricted water diffusion, $\boldsymbol{x}_{i}^{r}$ :

$$
\begin{gathered}
\nu^{\prime}=\frac{\sum_{i=1}^{M} \sum_{j=1}^{N_{r}} \boldsymbol{x}_{i_{j}}^{r}}{\sum_{i=1}^{M}\left(\sum_{j=1}^{N_{r}} \boldsymbol{x}_{i_{j}}^{r}+\sum_{j=1}^{N_{h}} \boldsymbol{x}_{i_{j}}^{h}\right)} \\
a_{i}^{\prime}=\frac{\sum_{j=1}^{N_{r}} 2 R_{j} \boldsymbol{x}_{i_{j}}^{r}}{\sum_{j=1}^{N_{r}} \boldsymbol{x}_{i_{j}}^{r}},
\end{gathered}
$$

where $R_{j}, j \in\{1, \ldots, N r\}$ denote the radius of the cylinder corresponding to the $j$-th atom in $\Phi_{i}^{r}$.

Hereafter, to make results easier to interpret for the reader, we refer to the original AMICO formulation [16] as $\mathrm{AMICO}_{1}$ and to its extended version for multiple fibres as $\mathrm{AMICO}_{\mathrm{x}}$. In the next section, the performance of both formulations is compared through numerical simulations.

\section{NUMERICAL SIMULATIONS}

To evaluate the effectiveness of $\mathrm{AMICO}_{\mathrm{x}}$, we tested it on synthetic data generated using the Monte-Carlo diffusion simulator system available in Camino [18], with the imaging protocol corresponding to a gradient strength $G \max =140 \mathrm{mT} / \mathrm{m}$ with 270 measurements divided into 3 shells with $b$-values $=\{1930,3090,13190\} \mathrm{s} / \mathrm{mm}^{2}$, corresponding to $G=\{140,131,140\} \mathrm{mT} / \mathrm{m}, \delta=\{10.2,7.6,17.7\}$ $\mathrm{ms}, \Delta=\{16.7,45.9,35.8\} \mathrm{ms}$ and same $T R / T E=5000 / 60 \mathrm{~ms}$ for all images. In all experiments when building the linear operators, we considered $N_{r}=10$ different axon radii in a range of 0.1 $8.5 \mu \mathrm{m}$, and $N_{h}=7$ different hindered environments corresponding to intra-axonal volume fractions from 0.3 to 0.9 . The regularisation parameter $\lambda$ was fixed to 0.25 .

We first simulated raw voxels with two fibre populations crossing at different angles (from $30^{\circ}$ to $90^{\circ}$ ). Each fibre population consisted of a distribution of different axon diameter, as done in [11], and several WM substrates were tested. For each configuration, different relative ratios of the two populations were evaluated. In each case, the mean and standard deviation of the estimated microstructural parameters was computed over 1000 repetitions, contaminating the signal with independent Rician noise realisations corresponding to $\mathrm{SNR}=30$, and compared them to the ground-truth. The estimation of the fibre orientations was performed using standard CSD [7] and the CSD peak estimation using the toolbox MRTrix ${ }^{1}$ with 90 measurements corresponding to the outer shell. For compactness, only results corresponding to relative volume fractions $f_{r_{1}}=$ $\{0.5,0.5\}$ and fibre population with 2 different radii - gamma distributions with parameters $\left(3.27,4.9 \cdot 10^{-7}\right)$ and $\left(4.82,2.6 \cdot 10^{-7}\right)$, respectively - corresponding to average axon diameters about 5.6 and

\footnotetext{
${ }^{1}$ http://www.nitrc.org/projects/mrtrix/
} 

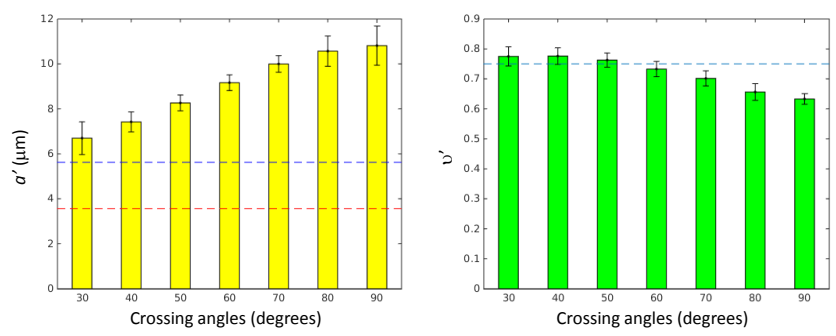

Fig. 1. Performance of $\mathrm{AMICO}_{1}$ on 2-fibre synthetic substrates as a function of the crossing angle between the fibres. Plots show the mean and standard deviation of the estimated mean axonal diameter (left) and intra-axonal volume fraction (right). Dashed lines represent the ground-truth values for the two populations.

3.6 micrometers are reported here. Results on the other substrates are consistent.

As a comparison, we fitted $\mathrm{AMICO}_{1}$ in the experimental settings described above to assess the impact of using this single-fibre model in regions with more than one fibre population. In these experiments, the atoms of the dictionary were oriented in the direction estimated with DTI, as in the original formulation [16]. The estimated microstructure indices (mean axon diameter and intra-cellular volume fraction) are compared with the ground-truth in figure 1. $\mathrm{AMICO}_{1}$ assumes that the fibres inside the voxel follow only one direction. Results show that making such an assumption in voxels that actually contain more than one fibre leads to erroneous estimation of microstructural properties. Mean axonal diameter appears overestimated whereas the intra-axonal volume fraction is underestimated; and the absolute error increases with the crossing angle of the ground-truth fibres. As expected, in the AMICO framework, the importance of correctly estimating the number of fibre bundles in order to choose a correct model appears to be crucial.

Figure 2 compares the microstructure parameters estimated from $\mathrm{AMICO}_{\mathrm{x}}$ with the ground-truth as a function of the crossing angles between the two fibre populations. The intra-cellular volume fraction can be estimated very accurately for all crossing angles (slightly over-estimated by about $4 \%$ ). The mean axonal diameter of the two fibre populations can be as well estimated pretty robustly (both slightly under-estimated) for all crossing angles. However, when the two orientations are too close $\left(\approx 30^{\circ}\right)$, the errors as well as the standard deviations of the estimates with respect to the ground-truth increase. These results are in line with (and slightly improve) those previously reported in [14]. The higher instability shown at $30^{\circ}$ can be well related to the performance of CSD in the peak-detection step. While the average angular error committed over the 1000 repetitions in crossings from $90^{\circ}$ to $40^{\circ}$ is less than $2^{\circ}$, CSD often identifies spurious peaks as true fibre directions for angles crossing at $30^{\circ}$, leading to a more unstable behaviour and higher average angular error.

Figure 3 illustrates the impact of the angular inaccuracy when estimating the orientation of the fibre populations, $\boldsymbol{\mu}_{i}$. In a substrate with two fibres crossing at a fixed angle of $60^{\circ}$, one of the directions used to build the dictionary was deviated from $1^{\circ}$ to $10^{\circ}$ from the actual orientation of the fibre. The intra-cellular volume fraction can be estimated accurately for all angular deviations, up to $10^{\circ}$. The estimation of the mean axonal diameter degrades progressively, yet absolute errors are smaller than $1 \mu \mathrm{m}$ for angular deviations up to $7^{\circ}$. These results are in-line with the angular accuracy of $\mathrm{AMICO}_{1}$ [16].

Lastly, the proposed model was tested also in a voxel with 3
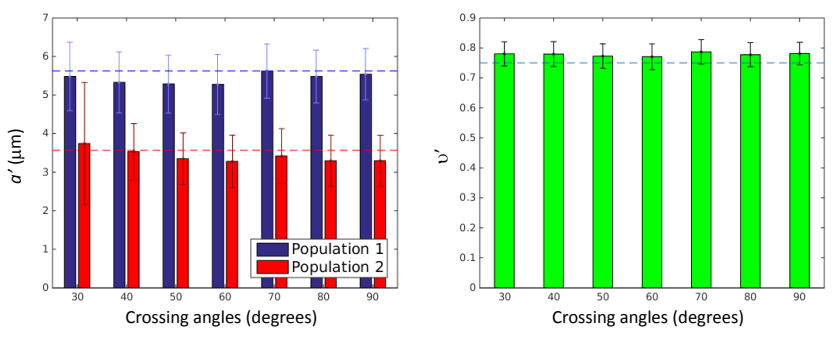

Fig. 2. Performance of $\mathrm{AMICO}_{\mathrm{x}}$ on 2-fibre synthetic substrates as a function of the crossing angle between the fibres. Plots show the mean and standard deviation of the estimated mean axonal diameter (left) and intra-axonal volume fraction (right) for the two different fibre populations. Dashed lines represent the ground-truth values for the two populations.
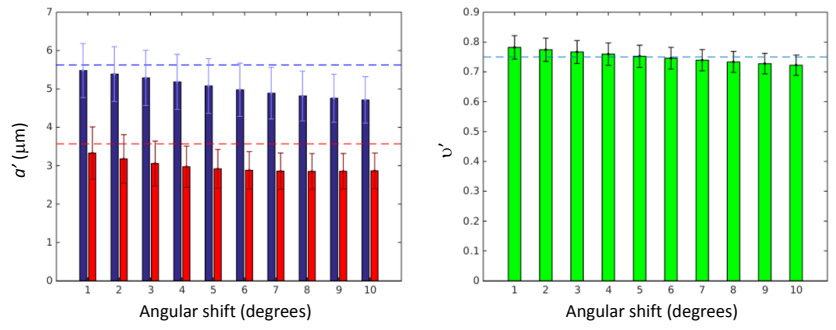

Fig. 3. Robustness to inaccuracies in the estimation of $\boldsymbol{\mu}_{2}$. Plots show the estimated mean axonal diameter (left) and intra-axonal volume fraction (right) as a function of the angular deviation of the estimated direction $\boldsymbol{\mu}_{2}$ with respect to the actual orientation of fibre population 2. Dashed lines represent the ground-truth values for the two populations.

non-coplanar fibre populations, as a proof of concept that evidences its generalisation to multiple fibre crossings. In this experiment, the crossing angle between two of the fibres was fixed to $90^{\circ}$ and the angle between the third one and the others varying between $30^{\circ}$ and $90^{\circ}$. Only results corresponding to a crossing of two populations with an average axon diameters about 5.6 and one of about $3.6 \mathrm{mi}-$ crometers are reported. Again, results with different substrates are consistent. Figure 4 compares the estimated microstructure features with the ground truth as a function of the crossing angles between the 3 estimated populations.

The non-optimised version of the code, implemented in MATLAB and run on a standard $2.70 \mathrm{GHz}$ Intel Core i7-3740QM processor, is able to fit the model in approximately $3.7 \mathrm{~ms} /$ voxel. Therefore, $\mathrm{AMICO}_{\mathrm{x}}$ still enables a drastic reduction of the computation time to solve the microstructure imaging problem as well in regions with multiple fibre populations compared to other non-linear routines, such as ActiveAx, which take $\approx 20$ s/voxel to fit its model [16].

\section{CONCLUSIONS}

In this paper, we have extented the original AMICO framework, that enables fast axonal diameter mapping with ActiveAx [11], to include crossing fibre populations within a voxel. Our results show through numerical simulations that $\mathrm{AMICO}_{\mathrm{x}}$ is indeed able to robustly estimate the microstructure parameters, provided the number and orien- 

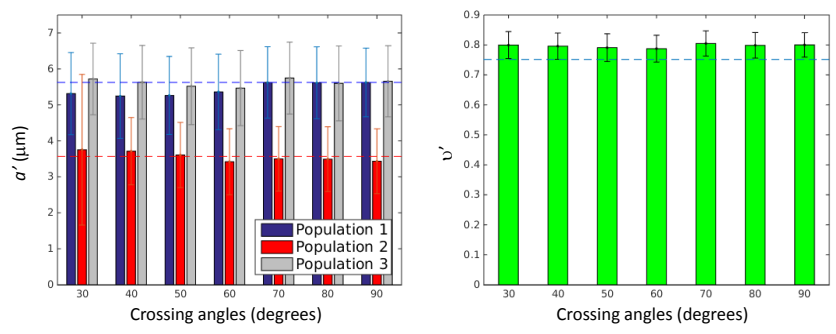

Fig. 4. Performance of $\mathrm{AMICO}_{\mathrm{x}}$ on 3-fibre synthetic substrates as a function of the crossing angle between the fibre population 1 and 2 . The crossing angle between populations 1 and 3 is fixed to $90^{\circ}$. Plots show the mean and standard deviation of the estimated mean axonal diameter (left) and intra-axonal volume fraction (right) for the three different fibre populations. Dashed lines represent the ground-truth values for the three populations.

tation of the fibre populations in a voxel is correctly estimated (up to $\approx 7^{\circ}$ of angular accuracy). We have as well shown how, thanks to the fast convex optimisation methods, $\mathrm{AMICO}_{\mathrm{x}}$ enables a reduction of the computation time by orders of magnitude with respect to other microstructure imaging techniques also in voxels with complex fibre configurations.

The extended data model of $\mathrm{AMICO}_{\mathrm{x}}$ can be fitted fast and accurately in all voxels of the brain, as allowed by [14], thanks to the generalisation of the original formulation to environments with multiple fibres. Moreover, in this framework, the coherence of the microstructural features among neighbouring voxels can be investigated and exploited using spatial regularisation terms that can be easily added to a convex formulation. Future work will be devoted to this study, including validation of the model on real data.

\section{REFERENCES}

[1] C. Beaulieu, "The basis of anisotropic water diffusion in the nervous system a technical review," NMR in Biomedicine, vol. 15, no. 7-8, pp. 435-455, 2002.

[2] PJ. Basser, J. Mattiello, and D. Le Bihan, "MR diffusion tensor spectroscopy and imaging," Biophysical journal, vol. 66, pp. 259-267, 1994.

[3] Van J. Wedeen, P. Hagmann, WY. Tseng, TG. Reese, and RM. Weisskoff, "Mapping complex tissue architecture with diffusion spectrum magnetic resonance imaging," Magnetic Ressonance in Medicine, vol. 54, pp. 1377-1386, 2005.

[4] E.Özarslan, T. M. Shepherd, B. C. Vemuri, S. J. Blackband, and T. H. Mareci, "Resolution of complex tissue microarchitecture using the diffusion orientation transform (DOT)," NeuroImage, vol. 31, pp. 1086-103, 2006.

[5] J.D. Tournier, F. Calamante, D. Gadian, and A. Connelly, "Direct estimation of the fiber orientation density function from diffusion-weighted MRI data using spherical deconvolution," NeuroImage, vol. 23, pp. 1176-1185, 2004.

[6] D.C. Alexander, "Maximum entropy spherical deconvolution for diffusion MRI," Information Processing in Medical Imaging (IPMI), pp. 76-87, 2005.

[7] J.D. Tournier, F. Calamante, and A. Connelly, "Robust determination of the fibre orientation distribution in diffusion MRI:
Non-negativity constrained super-resolved spherical deconvolution," NeuroImage, vol. 35, pp. 1459-1472, 2007.

[8] J.M. Ritchie, "On the relation between fibre diameter and conduction velocity in myelinated nerve fibres," Proc $R$ Soc Lond B Biol Sci., vol. 27, pp. 29-35, 1982.

[9] M. Shintaku, A. Hirano, and J.F. Llena, "Increased diameter of demyelinated axons in chronic multiple sclerosis of the spinal cord," Neuropathol. Appl. Neurobiol., vol. 14, pp. 505-510, 1982.

[10] E. Panagiotaki, T. Schneider, B. Siow, M. G. Hall, M. F. Lythgoe, and D. C. Alexander, "Compartment models of the diffusion MR signal in brain white matter: A taxonomy and comparison," Neurolmage, vol. 59, no. 3, pp. 2241-2254, 2012.

[11] D. C. Alexander, P. L. Hubbard, M. G. Hall, E. A. Moore, M. Ptito, G. J. M. Parker, and T. B. Dyrby, "Orientationally invariant indices of axon diameter and density from diffusion MRI.," NeuroImage, vol. 52, no. 4, pp. 1374-1389, 2010.

[12] T.B. Dyrby, L. V. SŁgaard, M. G. Hall, M. Ptito, and D.C. Alexander, "Contrast and stability of the axon diameter index from microstructure imaging with diffusion MRI," Magnetic Resonance in Medicine, vol. 70, no. 3, pp. 711-721, 2013.

[13] Y. Assaf and P. J. Basser, "Composite hindered and restricted model of diffusion (CHARMED) MR imaging of the human brain," NeuroImage, vol. 27, no. 1, pp. 48-58, 2005.

[14] H. Zhang, T.B. Dyrby, and D.C. Alexander, "Axon Diameter Mapping in Crossing Fibers with Diffusion MRI.," 2011, vol. 6892 of Lecture Notes in Computer Science, pp. 82-89, Springer.

[15] H. Zhang, T. Schneider, C. A. M. Wheeler-Kingshott, and D. C. Alexander, "NODDI: Practical in vivo neurite orientation dispersion and density imaging of the human brain.," NeuroImage, vol. 61, no. 4, pp. 1000-1016, 2012.

[16] A. Daducci, E.J. Canales-Rodriguez, H. Zhang, T.B. Dyrby, D.C. Alexander, and J.P. Thiran, "Accelerated Microstructure Imaging via Convex Optimization (AMICO) from diffusion MRI data," Neuroimage, vol. 105, pp. 32-44, 2015.

[17] P. L. Combettes and J.-C. Pesquet, "Proximal splitting methods in signal processing," Fixed-Point Algorithms for Inverse Problems in Science and Engineering (Bauschke H. H., Burachik R.S., Combettes P.L., Elser V., Luke D.R., Wolkowicz H., eds), pp. 185-212, 2011.

[18] M. G. Hall and D. C. Alexander, "Convergence and Parameter Choice for Monte-Carlo Simulations of Diffusion MRI.," IEEE Trans. Med. Imaging, vol. 28, no. 9, pp. 1354-1364, 2009. 